Check for updates

Cite this: Chem. Commun., 2022, 58,973

Received 23rd November 2021 Accepted 17th December 2021

DOI: $10.1039 / \mathrm{d} 1 \mathrm{cc} 06591 \mathrm{~b}$

rsc.li/chemcomm

\section{Metal-free transfer hydrochlorination of internal C-C triple bonds with a bicyclo[3.1.0]hexane- based surrogate releasing two molecules of hydrogen chloride $\dagger$}

\author{
Andreas J. Weidkamp (D) and Martin Oestreich (D)*
}

The development and application of a transfer hydrochlorination reagent based on a trichlorinated bicyclo[3.1.0]hexane core that transfers two molecules of $\mathrm{HCl}$ per molecule of surrogate to a $\pi$ basic substrate under $B\left(C_{6} F_{5}\right)_{3}$ catalysis is reported. Lewis acidassisted chloride abstraction followed by thermal electrocyclic cyclopropyl-to-allyl cation ring opening releases ring strain as a previously unexploited driving force.

Alkenyl chlorides are important building blocks in organic chemistry both on industrial and laboratory scale. For their synthesis, the addition of $\mathrm{HCl}$ across $\mathrm{C}-\mathrm{C}$ triple bonds is arguably the most atom-economic way of forming the $\mathrm{C}\left(\mathrm{sp}^{2}\right)$ $\mathrm{Cl}$ bond. However, this direct approach requires the use of corrosive and highly toxic $\mathrm{HCl}$ gas. Moreover, in the industrial benchmark process of hydrochlorination of acetylene to generate vinyl chloride, less appealing mercury-based catalysts are the state of the art although newer protocols using expensive palladium $^{-1}$ or gold-based ${ }^{2}$ catalysts have been developed. In more complex molecules, uncatalyzed, direct hydrochlorination with $\mathrm{HCl}$ is usually limited to electron-rich terminal alkynes, ${ }^{3}$ while ruthenium- $-{ }^{4}$ palladium- $-{ }^{5}$ platinum- $-{ }^{6}$ and gold-based ${ }^{7}$ catalysts have been shown to also convert a wider range of substrates including electron-deficient alkynes. ${ }^{8,9}$ Utilizing readily activated surrogates of commonly employed reagents can both solve handling problems and allow for the use of cheaper and less toxic catalysts albeit at the expense of reduced atom efficiency. Surrogates that contain more than one equivalent of the compound to transfer could be even more attractive but are currently not established.

A broadly applicable surrogate with reduced acidity compared to $\mathrm{HCl}$ was introduced by Hammond and $\mathrm{Xu}^{10}{ }^{10}$ Although a gold catalyst was typically required, a handful of more activated substrates reacted under metal-free conditions. Based

Institut für Chemie, Technische Universität Berlin, Strasse des 17. Juni 115, 10623 Berlin, Germany. E-mail: martin.oestreich@tu-berlin.de

$\dagger$ Electronic supplementary information (ESI) available: Experimental details and characterization data. See DOI: 10.1039/d1cc06591b on their research interest in transition-metal-catalyzed shuttle catalysis, ${ }^{11}$ Morandi and coworkers established non-acidic hydrochlorination surrogates. ${ }^{12}$ In a first protocol, 4chlorobutan-2-one was used as surrogate, yielding highly reactive methyl vinyl ketone as stoichiometric byproduct. While iridium catalysis was required, high functional-group tolerance was observed. Under more forcing conditions, the second protocol enabled the use of cheap tert-butyl chloride and liberated less reactive isobutene yet at the cost of a limited substrate scope.

A general strategy for ionic transfer hydrofunctionalizations employed by our group relies on the use of strong Brønsted or Lewis acids such as $\mathrm{B}\left(\mathrm{C}_{5} \mathrm{~F}_{6}\right)_{3}$ to activate otherwise bench-stable surrogates. ${ }^{13}$ Typically, these processes employ pro-aromatic reagents I passing through a reactive Wheland intermediate II before affording an aromatic byproduct III (Scheme 1, top). ${ }^{13,14}$ In this vein, we have successfully developed protocols for

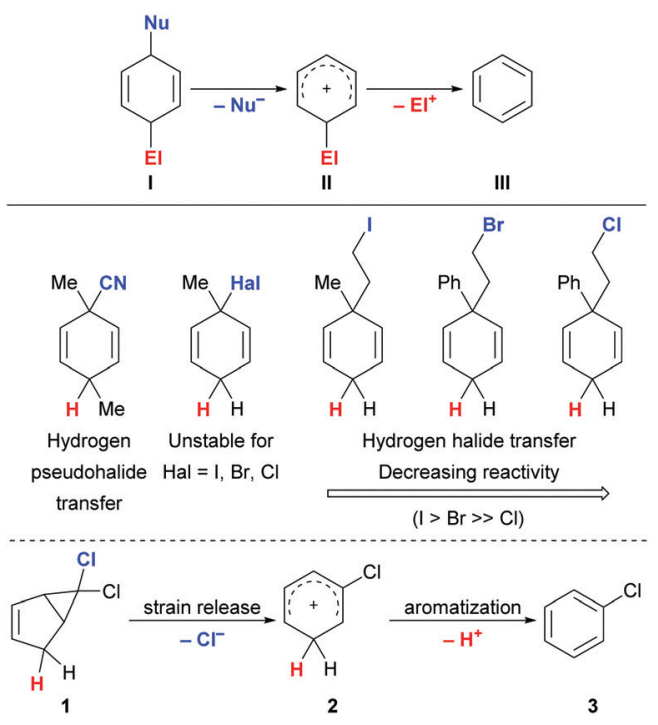

Scheme 1 Established hydrofunctionalization surrogates and development of a new surrogate for metal-free transfer hydrochlorination. 
transfer hydro(pseudo)halogenation such as transfer hydrocyanation, ${ }^{15}$-iodination ${ }^{16}$ and -bromination ${ }^{17}$ using surrogates that show no Brønsted acidity and do not possess protic hydrogen atoms (Scheme 1, middle). While for transfer hydrocyanation a simple 3-substituted cyclohexa-1,4-diene scaffold could be utilized, this strategy had to be refined for the transfer of $\mathrm{HI}$ and $\mathrm{HBr}$. In these cases, the corresponding 3halocyclohexa-1,4-dienes were not suitable as surrogates due to their chemical instability at room temperature. The solution that enabled the transfer of $\mathrm{HI}$ was the installation of an ethylene tether to stabilize the molecule that would eventually be cleaved to generate ethylene gas and the aforementioned Wheland intermediate. When adapting this concept to transfer hydrobromination, we realized that activation of the surrogate was the easier the weaker the $\mathrm{C}\left(\mathrm{sp}^{3}\right)-\mathrm{X}$ bond with $\mathrm{X}=\mathrm{I}$ and $\mathrm{Br}$ as well as $\mathrm{Cl}$. Overcoming this difficulty required higher temperatures and better stabilization of the intermediate carbenium ion with an additional phenyl group in the case of hydrobromination. These findings indicated that a transfer hydrohalogenation becomes increasingly complicated for the lighter halogen homologues and that a more sophisticated surrogate design might be necessary.

We commenced our quest for an $\mathrm{HCl}$ surrogate based on our experiences outlined above. Simple cyclohexa-1,4-diene surrogates were deemed as too unstable and, after preliminary experiments, we also learned that cleavage of the relatively strong $\mathrm{C}\left(\mathrm{sp}^{3}\right)-\mathrm{Cl}$ bond in ethylene-tethered surrogates was impossible at synthetically useful temperatures. ${ }^{18}$ We then devised the platform 1 for releasing the cationic key intermediate based on the thermal, disrotatory electrocyclic ring opening of bicyclo[3.1.0] hexanes (Scheme 1, bottom). ${ }^{19-21}$ After chloride abstraction, we intended to harness release of ring strain as a driving force for the generation of the desired Wheland complex 2. Subsequent aromatization to byproduct 3 would transfer the proton to the substrate. The parent system $\mathbf{1}$ is, however, described in the literature as thermally labile. ${ }^{19}$ For this reason, we considered and prepared the related surrogates 6-9 (Scheme 2, bottom) as potential alternatives.

Benzannulated surrogate $\mathbf{6}$ was obtained in one step from indene as a white solid in $40 \%$ yield by dichlorocarbene addition. $^{22}$ When employed in a test reaction with 1phenylprop-1-yne (4a) at $100{ }^{\circ} \mathrm{C}$ in $\mathrm{C}_{6} \mathrm{D}_{6}$ with $10 \mathrm{~mol} \%$ of $\mathrm{B}\left(\mathrm{C}_{6} \mathrm{~F}_{5}\right)_{3}$ as catalyst, we found the formation of the desired vinyl chloride 5a in 97\% NMR yield after $19 \mathrm{~h}$ (Scheme 2, top). Encouraged by this initial result, we envisioned that the double bond present in $\mathbf{1}$ could also be generated during the transfer reaction by another elimination of $\mathrm{HCl}$. A potential surrogate would thus carry two molecules of $\mathrm{HCl}$, thereby allowing for the transfer of both. Surrogate cis-7 bearing an additional chlorosubstituent on the cyclopentane ring was prepared and tested in the model reaction. ${ }^{23}$ Surprisingly, no product formation could be detected even after prolonged reaction time. The independently tested diastereomer trans-7 was equally unreactive, indicating that it is not steric hindrance imposed by that chlorine atom that inhibits the reaction. We hypothesized that the activation of the surrogate is facilitated by better
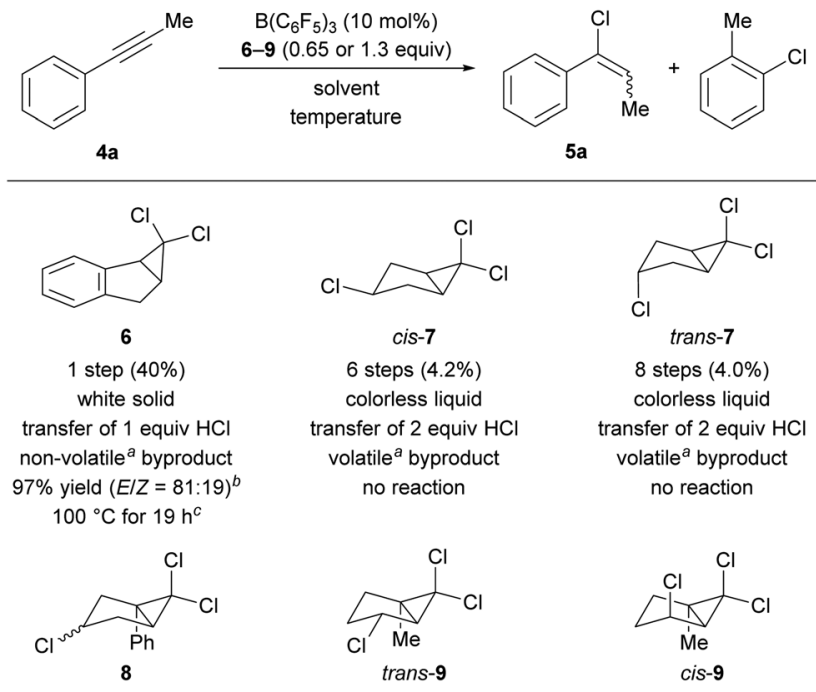

$$
7 \text { steps }(1.2 \%)
$$$$
\text { white solid }
$$

transfer of 2 equiv $\mathrm{HCl}$ non-volatile ${ }^{a}$ byproduct

$67 \%$ yield $(E / Z=74: 26)^{b}$ $140{ }^{\circ} \mathrm{C}$ for $80 \mathrm{~h}^{\mathrm{C}}$

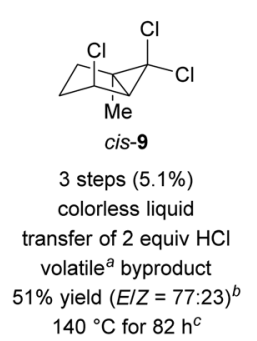

Scheme 2 Developed surrogates and their properties. ${ }^{a}$ Volatile was arbitrarily defined as having a boiling point below $160^{\circ} \mathrm{C} .{ }^{b}$ Yield and d.r. obtained in the model reaction were determined by ${ }^{1} \mathrm{H}$ NMR spectroscopy with dimethyl terephthalate as an internal standard. ${ }^{c}$ Reactions at $100{ }^{\circ} \mathrm{C}$ and $140{ }^{\circ} \mathrm{C}$ were run in $\mathrm{C}_{6} \mathrm{D}_{6}$ and $\mathrm{C}_{6} \mathrm{D}_{5} \mathrm{Cl}$, respectively.

stabilization of the carbenium ion intermediate. We therefore prepared the phenyl-substituted surrogate 8, available in seven steps from furfuryl alcohol in $1.2 \%$ overall yield. Indeed, this surrogate successfully transferred two molecules of $\mathrm{HCl}$. The reaction time was significantly longer than that seen with surrogate 6. We attribute this to differences in orbital overlap of the aromatic system with the carbenium ion. On the one hand, the rigid geometry of $\mathbf{6}$ enforces a planar benzyl cation. Orbitals are then perfectly aligned for carbenium ion stabilization by the phenyl ring and the adjacent double bond. On the other hand, the phenyl ring in the cation derived from 8 can twist out of plane to avoid steric hindrance. This renders a system that rather resembles a mere allyl cation. In contrast, previous transfer-hydrobromination surrogates benefitted from installation of a phenyl group. ${ }^{17}$ As a general disadvantage of the productive surrogates $\mathbf{6}$ and $\mathbf{8}$, the byproducts 2 chloronaphthalene and -biphenyl are difficult to remove, and the latter is assumed to be highly toxic.

To provide carbeniumion stabilization without introducing a "heavy" phenyl group, we considered two effects: (1) a methyl group at the bridgehead position should lend further stabilization and (2) an adequately positioned chlorine atom could allow for the formation of a chloronium ion. ${ }^{24}$ We found that we could test the influence of both effects with the trichloroalkane trans-9. The requisite cis-configured alcohol for its synthesis was described in the literature as a rare example where a hydroxy function acts as a directing group for dichlorocarbene addition. ${ }^{25}$ Whereas any of the previous syntheses did 
not tolerate unprotected alcohols, a straightforward three-step synthesis was possible in this case (see the ESI $\uparrow$ for details). Having established the synthetic access, we investigated its reactivity in our model transfer hydrochlorination. Importantly, even 0.65 equivalents of the surrogate allowed for a yield of $76 \%$ of $5 \mathrm{a}$ after $7 \mathrm{~h}$ at $140{ }^{\circ} \mathrm{C}$. This confirmed the planned transfer of two molecules of $\mathrm{HCl}$ from one molecule of the surrogate. The lower yield compared to that obtained with surrogate 6 is counterbalanced by the unprecedented two-fold transfer and the generation of a harmless, volatile byproduct. As before, we were curious about the influence of the surrogate's relative stereochemistry and prepared cis-9. In the model reaction, we observed substantially lower reaction rate, and the product was obtained in lower yield ( $51 \%$ vs. $76 \%$ ). This could be due to increased steric hindrance around the chlorine atoms in cis-9 compared to the situation in trans-9. For optimal transfer efficiency, diastereopure trans-9 was therefore used in all following experiments.

We next turned to the optimization of the reaction parameters. The need for an unreactive, high-boiling, and polar solvent left little room for variation. With the surrogate and the reaction temperature largely set, we thus focused on testing different catalysts in chlorobenzene at $130{ }^{\circ} \mathrm{C}$ (Table 1). The weak boron Lewis acid $\mathrm{BPh}_{3}$ failed to promote the reaction (entry 2). $\mathrm{B}\left(\mathrm{C}_{6} \mathrm{Cl}_{5}\right)_{3}$, considered a stronger Lewis acid than $\mathrm{B}\left(\mathrm{C}_{6} \mathrm{~F}_{5}\right)_{3}$, was also ineffective, probably due to the increased steric hindrance around the boron center (entry 3). The Brønsted acid $\mathrm{Tf}_{2} \mathrm{NH}$, successfully applied to hydrobromination and -iodination, ${ }^{16,17}$ led to decomposition (entry 4). This finding points to the role of $\mathrm{B}\left(\mathrm{C}_{6} \mathrm{~F}_{5}\right)_{3}$ as a catalyst instead of being a simple initiator. ${ }^{26}$ As expected, no conversion of the substrate was detected in the absence of catalyst (entry 5). An increased catalyst loading of $\mathrm{B}\left(\mathrm{C}_{6} \mathrm{~F}_{5}\right)_{3}$ accelerated the reaction but was detrimental to the yield (entry $6 v s$. entry 1 ). Increasing the reaction temperature from $130{ }^{\circ} \mathrm{C}$ to $140{ }^{\circ} \mathrm{C}$ drastically reduced the reaction time and further improved the yield to $76 \%$ (entry 7 vs. entry 1).

We then set out to explore the substrate scope (Scheme 3, top). Although initially optimized for the hydrochlorination of unfunctionalized 1-phenylprop-1-yne (4a), we quickly found

Table 1 Selected examples of the optimization of the transfer hydrochlorination of an internal C-C triple bond $(\mathbf{4 a} \rightarrow \mathbf{5} \mathbf{a} \text {; } c \text {. Scheme } 2 \text {, top })^{a}$

\begin{tabular}{lllll}
\hline Entry & Catalyst $(\mathrm{mol} \%)$ & Time $(\mathrm{h})$ & d.r. $^{b}(E: Z)$ & Yield $^{b}(\%)$ \\
\hline 1 & $\mathrm{~B}\left(\mathrm{C}_{6} \mathrm{~F}_{5}\right)_{3}(10 \mathrm{~mol} \%)$ & 83 & $74: 26$ & 72 \\
2 & $\mathrm{BPh}_{3}(10 \mathrm{~mol} \%)$ & - & - & $<5$ (Conv) \\
3 & $\mathrm{~B}\left(\mathrm{C}_{6} \mathrm{Cl}_{5}\right)_{3}(10 \mathrm{~mol} \%)$ & - & - & $<5$ (Conv) \\
4 & $\mathrm{Tf}_{2} \mathrm{NH}(10 \mathrm{~mol} \%)$ & - & - & Decomp \\
5 & None & - & - & $<5$ (Conv) \\
6 & $\mathrm{~B}\left(\mathrm{C}_{6} \mathrm{~F}_{5}\right)_{3}(20 \mathrm{~mol} \%)$ & 2 & $74: 26$ & 65 \\
$7^{c}$ & $\mathrm{~B}\left(\mathrm{C}_{6} \mathrm{~F}_{5}\right)_{3}(10 \mathrm{~mol} \%)$ & 7 & $74: 26$ & 76
\end{tabular}

${ }^{a}$ Unless otherwise noted, all reactions were performed in $\mathrm{C}_{6} \mathrm{D}_{5} \mathrm{Cl}$ at $130{ }^{\circ} \mathrm{C}$ using surrogate trans-9 (0.65 equiv.). ${ }^{b}$ Diastereomeric ratio, yield, and conversion were determined by ${ }^{1} \mathrm{H}$ NMR spectroscopy with dimethyl terephthalate as an internal standard. ${ }^{c}$ Reaction performed at $140{ }^{\circ} \mathrm{C}$.
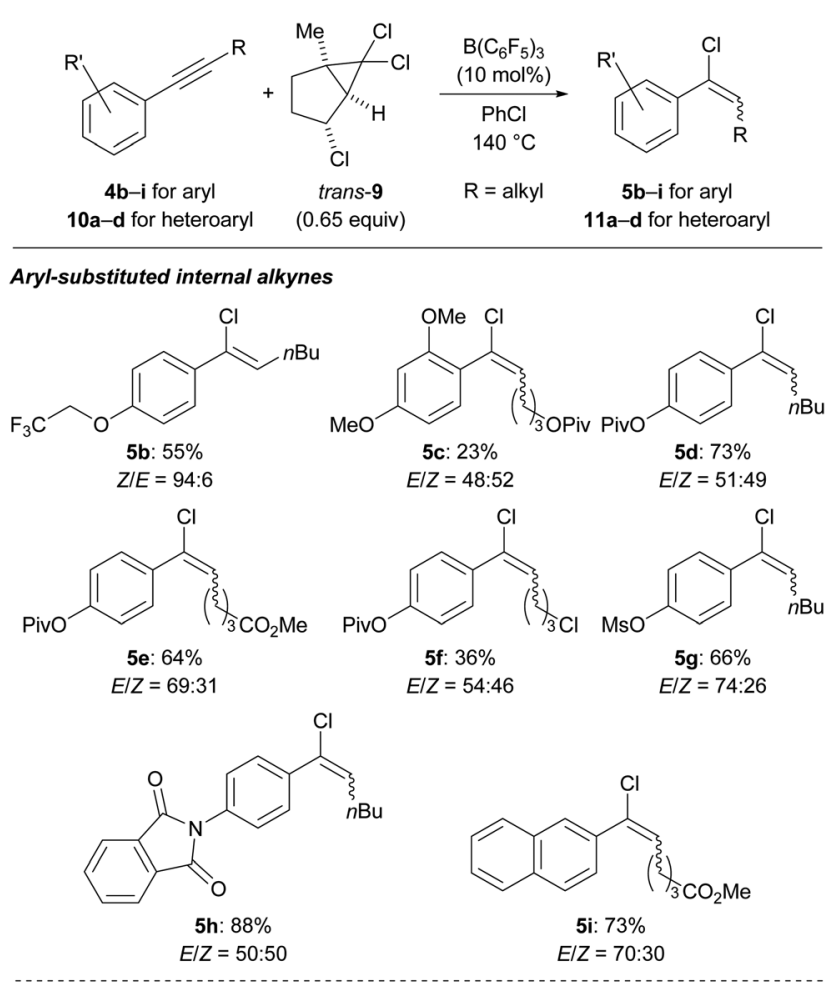

Heteroaryl-substituted internal alkynes
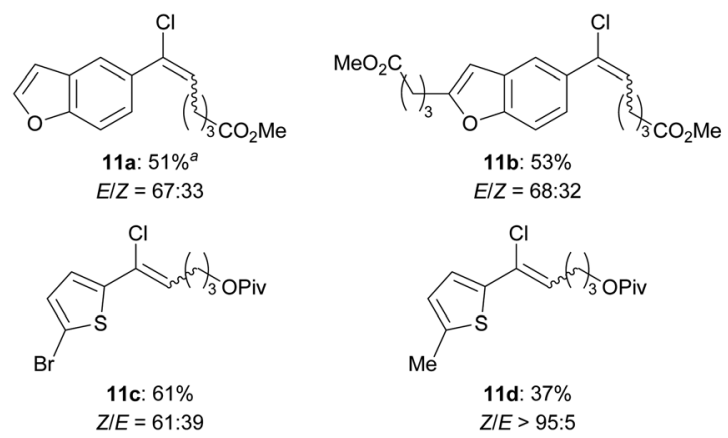

Scheme 3 Substrate scope of aryl/heteroaryl-substituted internal alkynes suitable for the transfer hydrochlorination. ${ }^{a} 74 \%(E / Z=71: 29)$ were obtained on a $2.0 \mathrm{mmol}$ scale.

that polar functional groups are tolerated. Their presence also made the chromatographic separation of the alkenyl chlorides from generated side products easier. As expected, arylsubstituted internal alkynes not having electron-withdrawing groups at the aryl ring participated in this ionic process. Various phenol derivatives $\mathbf{4} \mathbf{b}-\mathbf{g}$ either as an ether, ester or sulfonate reacted in good yields; the dimethoxyaryl group in $\mathbf{4 c}$ is likely too electron-rich for the acidic reaction medium. Substrate 4e containing two carboxyl groups was also amenable. A primary alkyl chloride as in $\mathbf{4 f}$ resulted in significant decomposition. A phthalimide group as in $\mathbf{4 h}$ was compatible, furnishing the desired vinyl chloride in high yield. The naphth2-yl-substituted alkyne 4i was converted into the product in good yield, while a similar substrate having a naphth-1-yl group was found to decompose under the reaction conditions (see the ESI $\dagger$ for details). 
We next investigated a variety of heteroaryl groups, potentially not compatible with $\mathrm{B}\left(\mathrm{C}_{6} \mathrm{~F}_{5}\right)_{3}$ at high temperature (Scheme 3, bottom). Notably, benzofurans turned out to be suitable substrates as exemplified by 10a and the 2-substituted derivative 10b. The reaction of a furyl-substituted derivative was successful but gave an unstable product that could not be isolated (see the ESI $\dagger$ for details). Thiophene derivatives such as 10c and 10d underwent the hydrochlorination without any problems.

The stereoselectivity of the reaction was generally modest, with the $E / Z$ ratio ranging from $74: 26$ to $39: 61$ in most cases. The obtained major isomer was normally $E$-configured which is in accordance with a vinyl cation as an intermediate accepting a chloride anion from the less hindered side either from the in situ-formed borate $\left[\mathrm{ClB}\left(\mathrm{C}_{6} \mathrm{~F}_{5}\right)_{3}\right]^{-}$or the surrogate itself. ${ }^{27}$

The present work is conceptual rather than being an advance in synthetic methodology. We have described a design for storable surrogates of $\mathrm{HCl}$ as an alternative to unstable or unreactive cyclohexa-1,4-diene-based platforms. Aromatization of the multiply chlorinated bicyclo[3.1.0]hexanes can be initiated by $\mathrm{B}\left(\mathrm{C}_{6} \mathrm{~F}_{5}\right)_{3}$-mediated chloride abstraction from a geminally dichlorinated cyclopropane unit. An electrocyclic ring opening driven by the release of strain followed by loss of a proton leads to a chlorobenzene byproduct with low molecular weight. The surrogate can be designed in such a way that another elimination of $\mathrm{HCl}$ is required to achieve aromatization. By this, two molecules of $\mathrm{HCl}$ can be generated from one molecule of the surrogate. With an internal $\mathrm{C}-\mathrm{C}$ triple bond as a proton acceptor, ${ }^{28}$ a $\mathrm{B}\left(\mathrm{C}_{6} \mathrm{~F}_{5}\right)_{3}$-catalyzed transfer hydrochlorination has become possible in moderate yields.

A. J. W. gratefully acknowledges the Berlin Graduate School of Natural Sciences and Engineering for a predoctoral fellowship (2017-2020). M. O. is indebted to the Einstein Foundation Berlin for an endowed professorship. We thank Markus Budde for his experimental contributions as well as Dr Weiqiang Chen and Pedro Helou de Oliveira for helpful discussions (all TU Berlin).

\section{Conflicts of interest}

There are no conflicts to declare.

\section{Notes and references}

1 J. Zhao, Y. Yue, G. Sheng, B. Wang, H. Lai, S. Di, Y. Zhai, L. Guo and X. Li, Chem. Eng. J., 2019, 360, 38-46.

2 J. Zhao, B. Wang, X. Xu, Y. Yu, S. Di, H. Xu, Y. Zhai, H. He, L. Guo, Z. Pan and X. Li, J. Catal., 2017, 350, 149-158.

3 (a) P. J. Kropp and S. D. Crawford, J. Org. Chem., 1994, 59, 3102-3112; (b) C.-X. Xu, C.-H. Ma, F.-R. Xiao, H.-W. Chen and B. Dai, Chin. Chem. Lett., 2016, 27, 1683-1685.

4 (a) H. Klein, T. Roisnel, C. Bruneau and S. Dérien, Chem. Commun., 2012, 48, 11032-11034; (b) S. Dérien, H. Klein and C. Bruneau, Angew. Chem., Int. Ed., 2015, 54, 12112-12115.

5 (a) J. Dupont, N. R. Basso, M. R. Meneghetti, R. A. Konrath, R. Burrow and M. Horner, Organometallics, 1997, 16, 2386-2391; (b) G. Zhu, D. Chen, Y. Wang and R. Zheng, Chem. Commun., 2012, 48, 5796-5798; (c) J. Derosa, A. L. Cantu, M. N. Boulous, M. L. O'Duill, J. L. Turnbull, Z. Liu, D. M. de La Torre and K. M. Engle, J. Am. Chem. Soc., 2017, 139, 5183-5193; (d) D. A. Petrone, I. Franzoni, J. Ye, J. F. Rodríguez, A. I. PobladorBahamonde and M. Lautens, J. Am. Chem. Soc., 2017, 139, 3546-3557.

6 C.-Y. Lo, M. P. Kumar, H.-K. Chang, S.-F. Lush and R.-S. Liu, J. Org. Chem., 2005, 70, 10482-10487.

7 J. Oliver-Meseguer, A. Doménech-Carbó, M. Boronat, A. Leyva-Pérez and A. Corma, Angew. Chem., Int. Ed., 2017, 56, 6435-6439.

8 For a review on transition-metal-catalyzed carbon-halogen bond formation, see: D. A. Petrone, J. Ye and M. Lautens, Chem. Rev., 2016, 116, 8003-8104.

9 The uncatalyzed, Michael-type addition of $\mathrm{HCl}$ to electron-deficient alkynes is long known. See: (a) R. Friedrich, Justus Liebigs Ann. Chem., 1883, 219, 368-374; (b) A. Michael and H. Pendleton, J. Prakt. Chem., 1889, 40, 63-68.

10 (a) R. Ebule, S. Liang, G. B. Hammond and B. Xu, ACS Catal., 2017, 7, 6798-6801; (b) X. Zeng, Z. Lu, S. Liu, G. B. Hammond and B. Xu, J. Org. Chem., 2017, 82, 13179-13187; (c) S. Liang, G. B. Hammond and B. Xu, Green Chem., 2018, 20, 680-684; (d) X. Zeng, S. Liu, G. B. Hammond and B. Xu, ACS Catal., 2018, 8, 904-909.

11 For general aspects of shuttle catalysis, see: $(a)$ B. N. Bhawal and B. Morandi, Angew. Chem., Int. Ed., 2019, 58, 10074-10103; for an aromatization-driven transfer dichlorination, see: $(b) \mathrm{X}$. Dong, J. L. Roeckl, S. R. Waldvogel and B. Morandi, Science, 2021, 371, 507-514.

12 P. Yu, A. Bismuto and B. Morandi, Angew. Chem., Int. Ed., 2020, 59, 2904-2910.

13 (a) J. C. L. Walker and M. Oestreich, Synlett, 2019, 30, 2216-2232; (b) S. Keess and M. Oestreich, Chem. Sci., 2017, 8, 4688-4695.

14 For radical-based approaches, see: A. Bhunia and A. Studer, Chem, 2021, 7, 2060-2100.

15 P. Orecchia, W. Yuan and M. Oestreich, Angew. Chem., Int. Ed., 2019, 58, 3579-3583.

16 W. Chen, J. C. L. Walker and M. Oestreich, J. Am. Chem. Soc., 2019, 141, 1135-1140.

17 W. Chen and M. Oestreich, Org. Lett., 2019, 21, 4531-4534.

$18 \mathrm{H}$. Fang and M. Oestreich, Unpublished results, Technische Universität, Berlin, 2019.

19 M. S. Baird, D. G. Lindsay and C. B. Reese, J. Chem. Soc. C, 1969, 1173-1178.

20 For the electrocyclic ring opening of cyclopropyl cations, see: (a) R. B. Woodward and R. Hoffmann, J. Am. Chem. Soc., 1965, 87, 395-397; (b) P. v. R. Schleyer, T. M. Su, M. Saunders and J. C. Rosenfeld, J. Am. Chem. Soc., 1969, 91, 5174-5176; Orbital symmetry dictates that in the present system, the chloride ion is released from the axial (= "endo") position. This has been experimentally verified: (c) P. S. Skell and S. R. Sandler, J. Am. Chem. Soc., 1958, 80, 2024-2025; (d) M. S. Baird and C. B. Reese, Tetrahedron Lett., 1967, 8, 1379-1382.

21 For a similar approach exploiting the ring strain of pro-aromatic bicyclo[2.2.0]hexenes, see: B. Wu, J. Wang, X. Liu and R. Zhu, Nat. Commun., 2021, 12, 3680.

22 (a) W. E. Parham and H. E. Reiff, J. Am. Chem. Soc., 1955, 77, 1177-1178; (b) W. E. Parham, H. E. Reiff and P. Swartzentruber, J. Am. Chem. Soc., 1956, 78, 1437-1440; for a seminal report, see: (c) G. L. Ciamician and M. Dennstedt, Ber. Dtsch. Chem. Ges., 1881, 14, 1153-1163.

23 I. Fleming and E. J. Thomas, Tetrahedron, 1972, 28, 4989-5001.

24 (a) G. A. Olah and J. M. Bollinger, J. Am. Chem. Soc., 1967, 89, 4744-4752; (b) G. A. Olah, J. M. Bollinger and J. Brinich, J. Am. Chem. Soc., 1968, 90, 2587-2594.

25 (a) D. Seyferth and V. A. Mai, J. Am. Chem. Soc., 1970, 92, 7412-7424; (b) R. H. Ellison, J. Org. Chem., 1980, 45, 2509-2511.

26 S. Banerjee and K. Vanka, ACS Catal., 2018, 8, 6163-6176.

27 As exceptions, the electron-rich substrates $\mathbf{4 b}$ and 10d gave almost pure $Z$-isomers as products. Presumably, thermodynamic control favors isomerization to the $Z$ isomer. As a general trend, the stereoselectivity of the reaction was found to considerably vary with the alkyne substrate but to be almost independent of the used surrogate. This indicates that chloride is released from the surrogate before the $\mathrm{C}\left(\mathrm{sp}^{2}\right)-\mathrm{Cl}$ bond is formed. As an alternative explanation for the observed diastereomeric ratios, thermodynamic equilibration of the alkenyl chlorides cannot be ruled out.

28 Terminal alkynes decomposed and dialkyl-substituted internal alkynes did not react. 